\title{
La politique de restauration des peintures des musées nationaux (1930-1950)
}

\section{Fernando Suárez San Pablo}

\section{(QpenEdition}

\section{Journals}

Édition électronique

URL : http://journals.openedition.org/cel/283

DOI : $10.4000 /$ cel.283

ISSN : 2262-208X

\section{Éditeur}

École du Louvre

\section{Référence électronique}

Fernando Suárez San Pablo, «La politique de restauration des peintures des musées nationaux (1930-1950) », Les Cahiers de l'École du Louvre [En ligne], 7 | 2015, mis en ligne le 01 octobre 2015, consulté le 17 septembre 2019. URL : http://journals.openedition.org/cel/283 ; DOI : 10.4000/cel.283

\section{cc) (†) $\odot$}

Les Cahiers de l'École du Louvre sont mis à disposition selon les termes de la licence Creative Commons Attribution - Pas d'Utilisation Commerciale - Pas de Modification 4.0 International. 


\section{Cahiers de l'École du Louvre}

recherches en histoire de l'art, histoire des civilisations archéologie, anthropologie et muséologie

Numéro 7. Octobre 2015

La politique de restauration des peintures

des musées nationaux (1930-1950)

Fernando Suárez San Pablo

Article disponible en ligne à l'adresse :

http://www.ecoledulouvre.fr/cahiers-de-l-ecole-du-louvre/numero7octobre-2015/suarez.pdf

Pour citer cet article :

Fernando Suárez San Pablo, « La politique de restauration des peintures des musées nationaux (1930-1950) 》[ en ligne] n 7 , octobre 2015, p. 33 à 45.

\section{बcreative \\ (요 $\circledast$}

(c) École du Louvre

Cet article est mis à disposition selon les termes de la Licence Creative Commons Attribution - Pas d'utilisation commerciale - Pas de modification 3.0 non transposé. 


\section{Cahiers de l'École du Louvre recherches en histoire de l'art, histoire des civilisations archéologie, anthropologie et muséologie}

\section{Numéro 7. Octobre 2015}

\section{Sommaire}

\section{Éditorial}

\section{Étude}

Caroline van Eck, Miguel John Versluys, Pieter ter Keurs, Leiden University, Departments of Art History, Archaeology, Anthropology/National Museum of Antiquities (résumé et texte intégral en pdf)

The biography of cultures: style, objects and agency. Proposal for an interdisciplinary approach.

\section{Articles}

Camille Bourdiel, ancienne élève de l'École du Louvre, diplômée de $2^{\mathrm{e}}$ cycle (résumé et texte intégral en pdf)

Exposer la science dans l'après-guerre. Hommage à Léonard de Vinci et Rembrandt, étude photographique et radiographique

au laboratoire du musée du Louvre.

Fernando Suárez San Pablo, ancien élève de l'École du Louvre, diplômé de $2^{\mathrm{e}}$ cycle (résumé et texte intégral en pdf)

La politique de restauration

des peintures des musées nationaux (1930-1950).

Alexandra Buvignier-Legros, ancienne élève de l'École du Louvre, diplômée de $2^{\mathrm{e}}$ cycle (résumé et texte intégral en pdf)

Pascal Häusermann et le motel L'Eau vive : une conception d'avant-garde ?... p. 46-53

Agnès Gué, ancienne élève de l'École du Louvre, diplômée de $2^{\mathrm{e}}$ cycle (résumé et texte intégral en pdf)

Goya dans l'historiographie française du XIX siècle : images et textes.

p. $54-63$

Constance Desanti, ancienne élève de l'École du Louvre, diplômée de $2^{\mathrm{e}}$ cycle (résumé et texte intégral en pdf)

Lumière sur Eugène Martial Simas, décorateur oublié de la Belle Époque. ...p. 64-75

Clémentine Delplancq, ancienne élève de l'École du Louvre,

diplômée de $2^{\mathrm{e}}$ cycle (résumé et texte intégral en pdf)

Faire carrière à Paris : Armand Bloch (1866-1932) et la Franche-Comté,

l'importance du soutien régional pour les artistes au XIX siècle.

p. $76-85$

Claire Merleau-Ponty, consultante en muséologie, (résumé et texte intégral en pdf) Vous avez dit médiation?

p. $86-88$

Andréa Delaplace, ancienne élève de l'EHESS, diplômée de $2^{\mathrm{e}}$ cycle (résumé et texte intégral en pdf)

Un palais pour les immigrés? Le Musée de l'histoire de l'immigration à Paris :

une collection et un musée en devenir.

p. $89-99$

Joan Despéramont, ancienne élève de l'École du Louvre, diplômée de $2^{\text {e }}$ cycle (résumé et texte intégral en pdf)

Les Souffleurs d'images. Un concept développé

par le Centre de Recherche Théâtre-Handicap (CRTH).

p. $100-106$ 


\section{La politique de restauration des peintures des musées nationaux (1930-1950)}

\section{Fernando Suárez San Pablo}

Le développement de techniques d'analyse scientifique appliquées à la connaissance des œuvres, l'entrée des scientifiques dans les musées, l'évolution des filières de formation des restaurateurs et les questionnements autour de leur statut qui en découlent sont autant de facteurs témoignant des profondes mutations que la conservation-restauration de biens culturels a connu au cours de la première moitié $\mathrm{du} \mathrm{xx}^{\mathrm{e}}$ siècle. Comme Pierre Leveau a montré, l'émergence de cette discipline en France à la fin des années 1970 est le résultat d'un processus dont les origines peuvent être en partie décelées dans l'entre-deux-guerres ${ }^{1}$. C'est en effet en 1926 que l'Office international des musées (OIM) ${ }^{2}$ est créé pour fédérer les institutions muséales sur le plan international, ayant comme instrument de travail principal la revue Mouseion, éditée dès l'année suivante ${ }^{3}$. Très tôt, la conservation et la restauration du patrimoine se révèlent être parmi les principales préoccupations partagées par les membres de l'OIM. Ainsi, une première conférence internationale sur ce sujet se tient à Rome du 13 au 17 octobre $1930^{4}$, aboutissant, dans le domaine de la peinture en particulier, à la publication d'un Manuel de conservation et de restauration de peintures en $1939^{5}$.

C'est dans ce contexte qu'un atelier de restauration des peintures des musées nationaux est organisé en 1935. Installé dans des locaux du musée du Louvre, c'est ce musée qui absorbe, en raison d'une vaste campagne de restauration de ses collections de peinture, la majorité de son activité jusqu'au début des années 1950. Plusieurs études ont retracé la carrière au service des collections publiques françaises de la première génération de restaurateurs ayant intégré l'atelier ${ }^{6}$. Mais qu'en est-il du point de vue institutionnel ? Le but de cet article est d'aborder cet épisode de l'histoire de la restauration en plaçant l'atelier au centre de la réflexion, de manière à dégager les besoins qui ont présidé à sa création, les moyens mis en œuvre pour son activité, et les mutations produites par cette nouvelle organisation.

Il n'existe pas, avant 1935 , de service structuré pour assurer les travaux de restauration des peintures du musée du Louvre, marqués jusqu'alors par le manque de ressources et de personnel. En effet, il semble que depuis 1919, Lucien Aubert a été le seul restaurateur de couche picturale intervenant au département des Peintures ${ }^{7}$. Les procès-verbaux de la commission de restauration

L'auteur tient à remercier Clémence Raynaud pour son aide et ses suggestions.

1. Pierre Leveau, «Problèmes historiographiques de la conservation-restauration des biens culturels », Conservation et restauration des biens culturels, no 26, 2008.

2. En représentation des institutions muséales françaises, Jean Guiffrey, conservateur du département des Peintures du musée du Louvre, et Henri Verne, directeur des musées nationaux, siègent au comité de gestion de I'OIM.

3. Marie Caillot, La revue Mouseion (1927-1946): Les musées et la coopération culturelle internationale, thèse pour le diplôme d'archiviste paléographe, Paris, École nationale des chartes, dir. Jean-Michel Leniaud, 2011.

4. Irène Portal, La Conférence internationale de Rome pour l'étude des méthodes scientifiques appliquées à l'examen et à la conservation des œuvres d'art, octobre 1930: une étape fondamentale de la conservation-restauration moderne, mémoire de recherche de master 2 , Paris, Ecole du Louvre, dir. Brigitte Bourgeois, Mario Micheli, 2012.

5. Une édition préliminaire de ce manuel parut, en 1938, sous le titre de « La Conservation des peintures », dans la revue Mouseion, vol. 41-42.

6. Voir notamment : Isabelle Cabillic, Jean-Gabriel Goulinat, chef de I'atelier de restauration des peintures des musées nationaux, 1935-1971, mémoire de master 2, Paris, Université Paris IV, dir. Barthélémy Jobert, 2006 ; I. Cabillic, « Jean-Gabriel Goulinat, chef de l'atelier de restauration des peintures des musées nationaux (1935-1971) », Techné, ns27-28, pp. 92-98, 2008 ; Laure Daran, Georges Dominique Zezzos (1883-1959): Artiste-peintre et restaurateur des musées nationaux, mémoire d'étude, Paris, École du Louvre, dir. Nathalie Volle, I. Cabillic, 2008 ; Sarah Davrinche, Lucien Aubert (1893-1979) : restaurateur de couche picturale au Musée du Louvre, mémoire d'étude, Paris, École du Louvre, dir. N. Volle, I. Cabillic, 2008 ; Florence Lépine, Pierre Paulet (1894-1978), Restaurateur de peintures des musées nationaux, mémoire de master 2, Paris, Université Paris IV, dir. Barthélémy Jobert, N. Volle; Fernando Suárez San Pablo, Pierre Michel (1889-1969), Restaurateur de couche picturale des musées nationaux, mémoire d'étude, Paris, Ecole du Louvre, dir. I. Cabillic, François Mirambet, 2012.

7. Carton «Lucien Aubert», Curriculum Vitae de monsieur Lucien Aubert (s. d.), Centre de recherche et de restauration des musées de France (C2RMF). 
des musées nationaux témoignent de l'activité relativement faible qui caractérise cette période, souvent limitée à des interventions d'urgence. Du personnel non qualifié peut participer aux travaux de restauration. En 1931 par exemple, Lucien Aubert se fait assister de "deux ou trois gardiens parmi les plus intelligents et soigneux " pour nettoyer les tableaux du Louvre à la térébenthine et à l'eau', pratique qui est poursuivie jusqu'en $1938^{9}$.

\section{Premières tentatives et expériences, 1930-1933}

Dès son arrivée à la direction des musées nationaux en 1926, Henri Verne envisage un plan ambitieux de réaménagement du musée du Louvre ayant pour but de regrouper les collections de chaque département et d'adapter leur présentation aux principes muséographiques modernes ${ }^{10}$. L'année suivante, il charge Jean-François Cellerier, directeur du laboratoire d'essais du Conservatoire national des arts et métiers, de créer un laboratoire de recherches scientifiques pour le musée afin d'" effectuer des études de documentation, de technique et d'identification des tableaux et œuvres d'art ${ }^{11}$ ". Très tôt, les missions du laboratoire sont élargies lorsque les restaurateurs Jean-Gabriel Goulinat, membre de la commission de restauration depuis $1923^{12}$, et Jacques Maroger, s'associent aux travaux (fig. 1). À l'occasion de la Conférence internationale de Rome organisée par l'Office international des musées (OIM) en 1930, les représentants du laboratoire du Louvre présentent les résultats des premières expériences. Jean-Gabriel Goulinat expose les apports de la photographie, la radiographie et l'observation des tableaux sous rayons ultraviolets au travail du restaurateur ${ }^{13}$, alors que Jean-François Cellerier s'intéresse à la prévention de la dégradation des œuvres d'art par le contrôle de l'éclairage et des conditions thermohygrométriques des salles d'exposition ${ }^{14}$. Le succès de cette conférence, dont la présence de la délégation française a été relayée amplement par la presse ${ }^{15}$, vient s'ajouter au don de documentation et d'appareillage que les docteurs argentins Carlos Mainini et Fernando Perez avaient réalisé la même année au profit des musées nationaux ${ }^{16}$, aboutissant à la création officielle d'un laboratoire au musée du Louvre en décembre $1932^{17}$.

Parallèlement à ces expériences, Henri Verne entreprend la réforme de la commission de restauration des musées nationaux, tombée dans la léthargie depuis de longues années malgré des tentatives de renouveau en 1919 et $1922^{18}$. À sa demande, une nouvelle commission est nommée le 13 mars $1930^{19}$, dès lors

8. «Rapports de MM. Maroger, Goulinat, Aubert, Nicolle et de I'Office international des musées au sujet de la conservation et de la restauration des tableaux », lettre de Jean Guiffrey au directeur des musées nationaux, 9 février 1932, Archives des musées Nationaux (AMN), T16 1930-1932. 9. Elle est alors interdite à la demande de Germain Bazin, qui constate que les gardiens « essuient les tableaux avec un chiffon ou les époussettent au moyen d'un plumeau ». Note de Germain Bazin à Henri Verne, 25 août 1938, AMN, 6LL3 1937-1949 (m).

10. Henri Verne, «Projet de réorganisation du musée du Louvre », Mouseion, vol. 10, 1930, pp. $5-13 ; \mathrm{H}$. Verne, « Le plan d'extension et de regroupement méthodique des collections du musée du Louvre. Les travaux de 1927 à 1934 », Bulletin des musées de France, 6 année, $\mathrm{n}^{\circ} 1,1934$, pp. 1-18; Jacques Jaujard, «Les principes muséographiques de la réorganisation du Louvre », Mouseion, vol. 31-32, pp. 7-30; H. Verne, « Les nouvelles installations du Louvre. Les travaux de 1934 à 1936. », Bulletin des musées de France, 8e année, n 5, 1936, pp. 66-68.

11. H. Verne, « Un laboratoire de recherches scientifiques au musée du Louvre », Bulletin des musées de France, $1^{\mathrm{e}}$ année, no 8, 1929, pp. 173-174.

12. I. Cabillic, 2008, op. cit. note 6, p. 92.

13. Jean-Gabriel Goulinat, «L'apport des procédés scientifiques dans la restauration des peintures », Mouseion, vol. 15, 1931, pp. 47-53.

14. Jean-François Cellerier, «Le chauffage, la ventilation et l'éclairage dans les salles d'exposition », Mouseion, vol. 16, 1931, pp. 66-76.

15. Comme en témoigne une collection de coupures de presse conservée par le C2RMF, carton « GR ».

16. Voir une coupure de presse d'un article de Georges Brunon Guardia, « Le Don de M. et $M^{m e}$ Mainini aux musées nationaux », juillet 1930, conservé dans AMN, PL1.

17. Arrêté du 12 décembre 1932, AMN, PL1. Une commission d'experts avait été préalablement chargée de l'organisation du laboratoire. Arrêté du 20 mars 1930, AMN, PL1.

18. Rapport du Ministère des Beaux-Arts concernant la réorganisation de la restauration, 25 février 1919 et lettre du directeur des musées Nationaux au sous-secrétaire d'État des Beaux-Arts, 12 février 1930, AMN, P2R1A. Cité par Julie Chaizemartin, Isabelle Cabillic, « Les commissions de restauration des peintures des musées Nationaux de 1882 à 1937 », Technè, nos 27-28, 2008, pp. 158. Voir aussi Julie Chaizemartin, Les commissions consultatives des restaurations de 1882 à 1946 : finalité, mise en place, membres, organisation, œuvres étudiées er restaurées, mémoire d'étude, Paris, Ecole du Louvre, dir. Nathalie Volle, Isabelle Cabillic, 2005.

19. Arrêté du 13 mars 1930, AMN, P2R1A. 
onze membres représentant l'administration et seize personnalités invitées à titre particulier l'intègrent. La volonté de favoriser le débat et la critique indépendante se traduit par la nomination d'individus aux compétences très diverses. Ainsi, aux côtés du directeur du laboratoire Jean-François Cellerier et des restaurateurs Lucien Aubert, Jean-Gabriel Goulinat et Jacques Maroger, siègent des amateurs, des critiques d'art, des peintres et des graveurs.
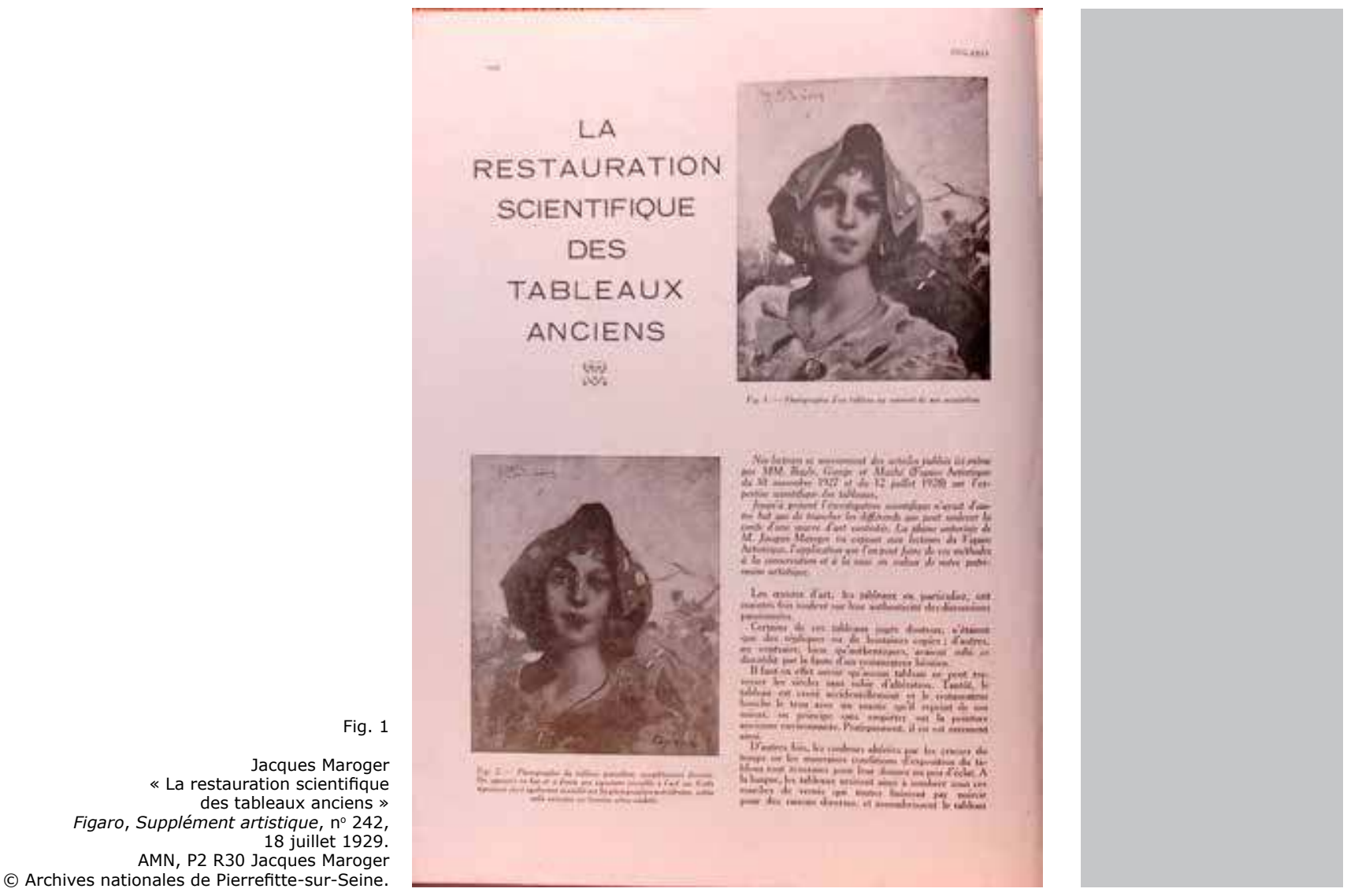

Quelques jours plus tard, la commission signale «l'impulsion à donner aux travaux de restauration ${ }^{20}$ ", qui devient rapidement un plan de nettoyage des peintures du musée du Louvre ${ }^{21}$. En février 1932, cependant, le sous-secrétaire d'État aux Beaux-Arts charge Henri Verne de mener une enquête sur les méthodes de nettoyage et de restauration employés au musée ${ }^{22}$. Henri Verne demande l'arrêt des travaux jusqu'à la fin de l'enquête, dont Jacques Maroger se trouve vraisemblablement à l'origine. En effet, celui-ci "incrimine, de façon générale ${ }^{23}$ " devant la commission le procédé de nettoyage des tableaux employé par Lucien Aubert, accusé de les laver à l'eau et au savon. Les deux rapports adressés par Maroger à Verne soulignent le manque d'organisation des travaux de restauration : "Tentatives isolées, sans plan préconçu, donc sans profit pour l'avenir », ainsi que les dysfonctionnements de la commission de restauration, dont il dénonce le temps insuffisant pour examiner les œuvres et le laconisme des procès-verbaux ${ }^{24}$.

20. Procès-verbal du 28 mars 1930, C2RMF. Les procès-verbaux des commissions de restauration sont conservés également aux AMN, série P2R1C.

21. Procès-verbal du 17 novembre 1930, C2RMF. « La Commission s'accorde sur la nécessité de nettoyer les tableaux, tout en insistant sur l'obligation de veiller à la limitation de cette opération. L'organisation d'un service de nettoyage est prévue. »

22. « Rapports de MM. Maroger, Goulinat, Aubert, Nicolle et de l'Office international des musées au sujet de la conservation et de la restauration des tableaux », minute de lettre du directeur des musées nationaux à J.-G. Goulinat, Marcel Nicolle, René Piot, Armand Point et Jacques Maroger, 3 février 1932, AMN, T16 1930-1932.

23. Procès-verbal du 16 mars 1932, C2RMF.

24. «Rapports de MM. Maroger, Goulinat, Aubert, Nicolle et de l'Office international des musées au sujet de la conservation et de la restauration des tableaux », J. Maroger, Notes sur la conservation et la restauration des tableaux, AMN, T16 1930-1932 et rapport adressé au directeur des musées nationaux concernant la restauration des tableaux du Louvre, 9 février 1932 ; rapport de $M$. Maroger sur les commissions de restauration des 26 juin 1930, 17 novembre 1930, 19 juin 1931, 10 février 1932, AMN, P2R30 Jacques Maroger. 
Il propose la réorganisation des travaux par la création d'un " conseil technique " qui étudierait, en collaboration avec le laboratoire, les tableaux proposés par les conservateurs et soumettrait un rapport détaillé aux membres de la commission avant leur restauration. Il envisage, enfin, l'établissement d'un dossier d'imagerie scientifique avant et après restauration, et la création d'une école de restauration au sein du musée.

Quelques idées communes peuvent être dégagées des rapports fournis par Jean Guiffrey, Lucien Aubert, Jean-Gabriel Goulinat, Jacques Maroger et Marcel Nicolle, pouvant servir à définir les principes de la restauration des peintures telle qu'elle est alors pratiquée au musée du Louvre ${ }^{25}:$ bien que chaque expert préconise des procédés différents, le nettoyage superficiel des peintures et la régénération des vernis sont jugés inoffensifs, quoique la portée de ces opérations doit être mesurée, ces tâches pouvant être confiées, selon certains avis, à des individus sans qualifications dans la restauration. Henri Verne souligne l'importance de l'étude du tableau avant toute intervention et la nécessité d'adapter les procédés de restauration aux problématiques particulières et non «de se rallier à tel ou tel système ${ }^{26}$ ". Au-delà de ces opérations, il est réservé à un restaurateur qualifié d'intervenir, qui doit se limiter à faire «l'indispensable, ne toucher à la peinture qu'en cas de nécessitée ${ }^{27}$ ".

La controverse arrive à sa fin le 18 avril 1932 lorsque la commission assiste aux essais de nettoyage sur La Vierge aux rochers de Léonard de Vinci (Inv. 777) ${ }^{28}$. Lucien Aubert montre son procédé qui consiste à frotter la surface du tableau à l'aide d'un tampon d'ouate imbibé d'essence de térébenthine et de quelques gouttes d'eau qu'il sèche ensuite avec des tampons secs, suivi d'une régénération du vernis à l'alcool. Le procédé est accepté «à l'unanimité " par la commission. Dès lors, l'accord sur les méthodes de nettoyage, quoique les pratiques aient évolué dans les années suivantes, et la définition des principes préfigurant une doctrine de la restauration des peintures, permet la poursuite des travaux avec un élan renouvelé.

\section{La définition du rôle de la commission de restauration}

Mais les campagnes de restauration se heurtent encore au manque de ressources financières à la fin de 1933 : « Nous attendons pour proposer de restaurer et de soigner 150 peintures malades, du Louvre, les crédits nécessaires ${ }^{29}$ ». Entretemps, à l'occasion des premiers travaux du plan Verne, les anciens ateliers de restauration de peinture sont transformés en salles d'exposition et de nouveaux locaux sont aménagés sous le comble sud-est de la Cour Carrée. Décrit par Jacques Jaujard, le nouvel atelier est " un local de $300 \mathrm{~m}^{2}$, bien éclairé par un vitrage supérieur et disposant de tous les moyens de travail modernes ${ }^{30} "$.

La commission de restauration des musées nationaux connaît des remaniements annuels entre 1934 et 1938. Ces flottements, liés à la réorganisation des travaux de restauration, peuvent être expliqués par la volonté de se doter d'un organe à double fonction - prise de décisions sur les choix des restaurations et approbation postérieure de ceux-ci - auquel siègent simultanément des spécialistes de la restauration et d'autres membres sans connaissance dans le domaine. En outre, la méfiance d'une partie de la critique et du public envers l'aspect des tableaux après avoir été nettoyés (comme l'illustre la vive polémique qui éclate dans la presse après la restauration réalisée par Jean-Gabriel Goulinat en 1935 du Jeune homme à la large toque noire, dit aussi Portrait de Titus, tenu à l'époque pour un Rembrandt,

25. Op. cit. notes 22 et 24.

26. Rapport de M. Henri Verne sur les Commissions de Restauration des séances des 16 mars et 18 avril 1932, AMN, P2R2.

27. « Rapports de MM. Maroger, Goulinat, Aubert, Nicolle et de l'Office international des musées au sujet de la conservation et de la restauration des tableaux $\gg$, rapport de Marcel Nicolle au directeur des musées nationaux, 7 mars 1932, AMN, T16 1930-1932.

28. Procès-verbal du 18 avril 1932, C2RMF.

29. Note pour Georges Huisman, directeur général des Beaux-Arts, 13 novembre 1933, C2RMF, Archives de la Restauration I (1/2), Atelier de restauration (1934- $1^{\text {er }}$ semestre 1939).

30. J. Jaujard, op. cit. note 10, p. 13 ; H. Verne, op. cit. note 10, 1934, p. 13. 
(Inv. 1749) ${ }^{31}$ ) amène Henri Verne à essayer de se servir de la commission comme instrument de légitimation de la nouvelle politique de nettoyages ${ }^{32}$.

Le 16 février 1935, la commission propose la création de deux souscommissions afin de coordonner les campagnes de restauration qui doivent commencer prochainement :

"Le rôle des Sous-commissions est d'examiner l'état des tableaux du Louvre et d'établir une liste des travaux à effectuer en tenant compte à la fois de leur degré d'urgence et de leur importance. Lorsque la restauration d'un tableau a été décidée, une étude préparatoire est faite au Laboratoire du Musée, qui établit un dossier de restauration comprenant outre une bonne photographie mettant en évidence les lésions de la peinture, une description de celle-ci et tous renseignements pouvant être utiles au restaurateur. Le traitement envisagé pour le tableau après entente avec les restaurateurs et avis de la Commission est porté au dossier ainsi que le travail effectué avec les diverses modifications qu'il y a lieu d'apporter au projet primitif et des photographies justificatives jusqu'à l'achèvement du travail ${ }^{33}$. "

La définition du contenu du dossier de restauration affirme l'importance accordée à la création d'une documentation ${ }^{34}$ dont la finalité est triple : assister les restaurateurs dans leur travail, informer la prise de décisions de la commission, et remplir une mission pédagogique auprès du public dans le but d'éviter des polémiques $^{35}$.

L'esprit de cette sous-commission dite "technique " dans laquelle siègent le directeur des musées nationaux, un membre de l'Institut, les conservateurs du Louvre et de Versailles, quatre restaurateurs et un scientifique ${ }^{36}$, est en effet de séparer les deux fonctions principales de la commission. Ainsi, les spécialistes décident des œuvres à restaurer et des opérations qui doivent être réalisées, tandis que la commission principale approuve leurs choix. C'est pourquoi le 19 janvier 1936, alors que la commission se dote, pour la première fois, d'un règlement définissant de manière spécifique ses attributions, les restaurateurs en sont exclus ${ }^{37}$ (fig. 2a et b).

Cependant, Jean-Gabriel Goulinat fait remarquer au directeur général des Beaux-Arts « le manque de liaison réel qui existe entre cette commission proprement dite ", dans laquelle "il n'y a pas un seul spécialiste de la restauration ${ }^{38}$ ", et la sous-commission technique, si bien que le 9 avril 1937 les restaurateurs regagnent leur place dans la commission. Ce n'est qu'après la Seconde Guerre mondiale que ces tentatives aboutissent à la mise en place d'une commission désormais professionnalisée où ne siègent que quatorze membres, tous expérimentés dans le domaine de la restauration.

\footnotetext{
31. Isabelle Cabillic, Béatrice Lauwick, «La restauration des Pèlerins d'Emmaüs en 1894 et du Portrait de jeune homme coiffé d'une large toque noire, dit Portrait de Titus, en 1935, ou les "affaires Rembrandt" », Technè, $\mathrm{n}^{\circ} 35,2012$, pp. 81-87; Pierre Alban Vinquant, L'Affaire du Titus : de la polémique suscitée par l'allégement de vernis à la remise en cause de l'attribution du Portrait de jeune homme à Rembrandt, mémoire de recherche, Paris, École du Louvre, dir. N. Volle, Clémence Raynaud 2005.

32. Lettre du directeur des musées nationaux au directeur général des Beaux-Arts, 4 mars 1935, AMN, P2R1A.

33. Procès-verbal de la commission du 16 février 1935, C2RMF. Cité par Clémence Raynaud, «Les archives de la restauration au Centre de recherche et de restauration des musées de France », Technè, nos 27-28, 2008, p. 43

34. Idem, Ibidem.

35. Comme l'illustre l'art. 6 de l'arrêté du 19 janvier 1936, AMN, P2R1A : « Les peintures entretenues ou restaurées, conformément aux avis de la commission, sont exposées pendant un mois [...]. À côté de ces peintures seront présentées les photographies ou documents essentiels pour apprécier les soins donnés et leur résultat. »

36. Procès-verbal de la sous-commission technique de la commission de restauration du 14 mai 1937, C2RMF.

37. Arrêté du 19 janvier 1936, AMN, P2R1A.

38. Lettre de Goulinat à Georges Huisman, 6 décembre 1936, AMN, P2R1A.
} 


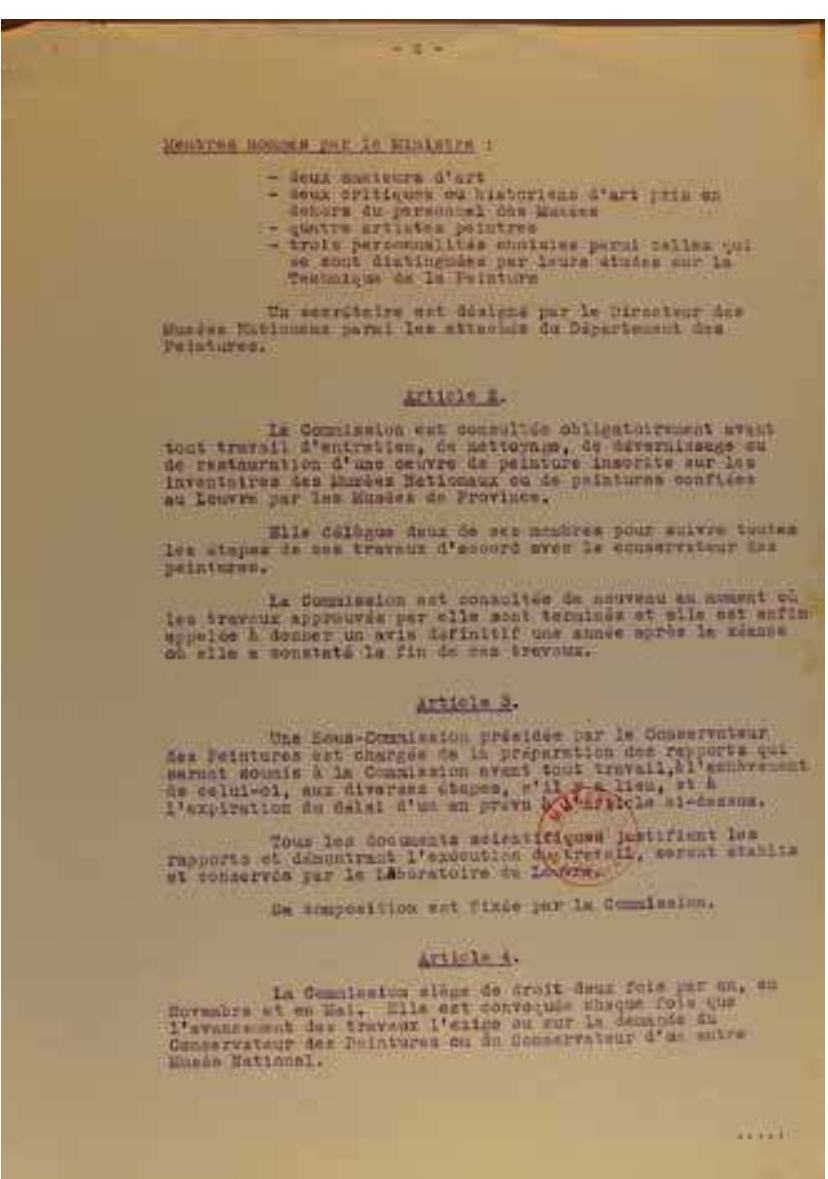

Fig. $2 a$ et $2 b$

Règlement de la commission de restauration des musées nationaux.

1936 du Ministère de

I'Education nationale.

$$
\text { AMN, P2R1A }
$$

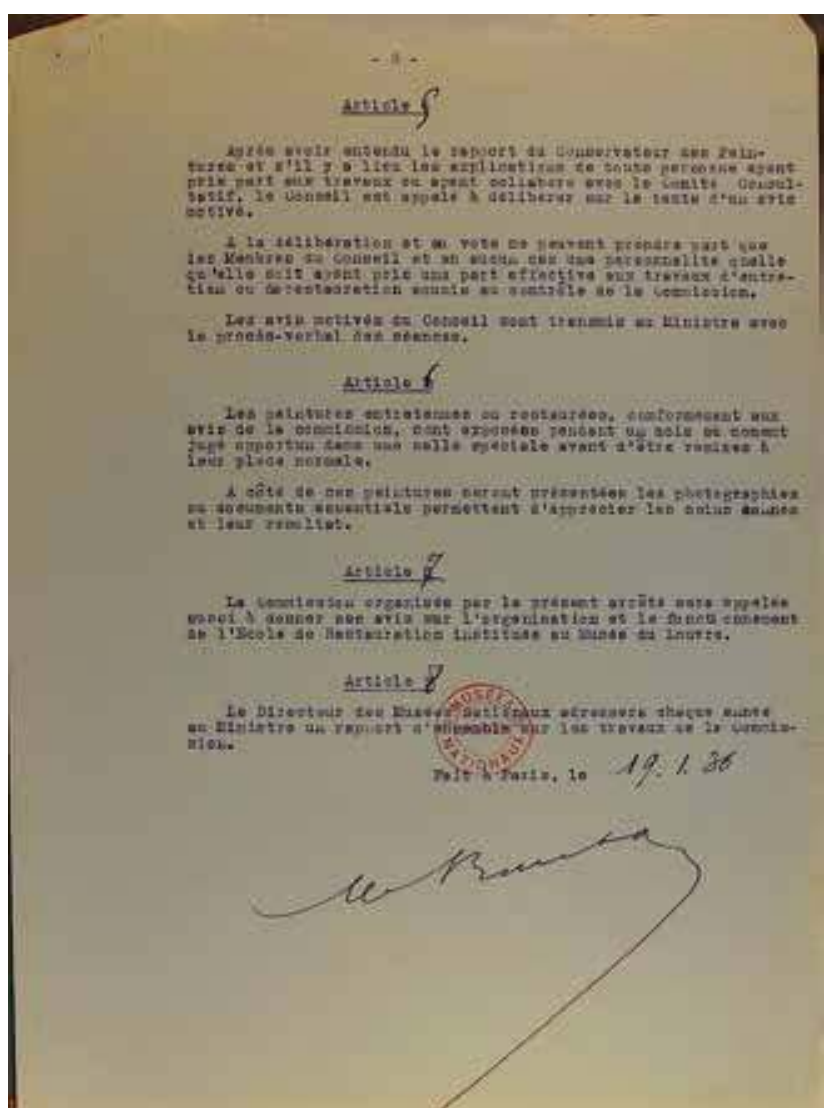


Bien que divers projets de création d'une école de restauration soient envisagés depuis $1932^{40}$, le besoin de commencer les campagnes de restauration conduit Henri Verne à demander au directeur général des Beaux-Arts la mise en place d'un concours de restaurateurs ${ }^{41}$ qu'il justifie, en réponse aux critiques formulées par certains syndicats, en évoquant la nécessité de contrôler les connaissances des candidats : "C'est que jusqu'ici la profession de restaurateur n'a jamais été l'objet d'un enseignement scientifique, mais d'un simple apprentissage pratique traditionnel ${ }^{42} »$.

Paul Jamot, conservateur du département des Peintures du musée du Louvre, préside le jury ${ }^{43}$ formé de Jacques Dupont, nommé directeur du laboratoire du musée en 1935, et des restaurateurs Jean-Gabriel Goulinat, Lucien Aubert, Jacques Maroger et Edgard Aillet, ce dernier nommé à la commission en $1934^{44}$. Les épreuves prévues, qui correspondent à celles proposées par Goulinat dans un Projet pour une École Nationale de Restauration de tableaux ${ }^{45}$ daté de 1934, mettent en lumière les qualités requises. Trente-trois candidats sont convoqués à la première épreuve le 29 juin 1935. Elle consiste à identifier l'époque, l'école et l'auteur de onze tableaux, puis à constater l'état de conservation des toiles et à proposer un traitement en détaillant les opérations envisagées ${ }^{46}$. Selon Henri Verne, en effet, "l'épreuve de restauration ne saurait être suffisante si d'abord celui qui y est soumis ne peut expliquer ce qu'il entend faire : opérer sans diagnostic ne saurait valoir, puisqu'il serait impossible, après le concours, de confier des chefs-d'œuvre à des peintres capables seulement d'une habileté pratique incontestable et avec qui on ne saurait s'entendre sur les soins à donner à une ouvre déterminée ${ }^{47}$ ". Le 4 juillet, dix candidats sont convoqués à l'épreuve pratique de dévernissage et de restauration ${ }^{48}$. S'agissant du dévernissage, les qualités appréciées sont l'assurance dans l'exécution et la maîtrise du processus. Parmi les candidats que le jury considère être "au courant des méthodes modernes ", on trouve l'emploi des solvants suivants, bien que chaque restaurateur les mélange différemment: xylol, essence de Myrlone, alcool, essence de térébenthine, essence d'aspic, acétone et toluène. Concernant la retouche, l'emploi de couleurs a tempera est exigé, l'harmonie des retouches et leur restriction aux limites des lacunes étant jugée ${ }^{49}$. Le 20 juillet se déroule la dernière épreuve, à laquelle quatre candidats sont convoqués ${ }^{50}$. Il s'agit de réaliser la copie d'un tableau du musée, examen qui suit la conception du métier de restaurateur de peintures de Jacques Maroger selon laquelle "la restauration est une branche spéciale de l'art. Son critérium

39. Le déroulement de ce concours a aussi été étudié par Béatrice Lauwick, Isabelle Cabillic, Claire Gerin-Pierre, «Les concours de restaurateurs de peinture au Louvre », Technè, n ${ }^{\circ s} 27-28$, 2008, pp. 108,109.

40. Pierre Leveau, op. cit. note 1, p. 14

41. Lettre du directeur des musées nationaux au directeur général des Beaux-Arts, 25 mars

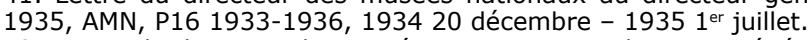

42. Lettre du directeur des musées nationaux au directeur général des Beaux-Arts, 9 décembre 1935, AMN, P2R1A ; lettre de Paul Mignon à Henri Verne, 29 juin 1935, AMN, O5E, Convocation des candidats au concours des restaurateurs.

43. Lettre du directeur des musées nationaux à Paul Jamot, 18 juillet 1935, AMN, O5E, $2^{\mathrm{e}}$ partie du Concours des restaurateurs.

44. Convocation du jury du Concours des restaurateurs, 17 juin 1935, AMN, O5E ; arrêté du 23 janvier 1934, AMN, P2R1A.

45. AMN, P16 1933-1936. Cité par I. Cabillic, 2008, op. cit. note 6, p. 93.

46. $1^{\text {re }}$ partie du Concours des restaurateurs, AMN, O5E.

47. Lettre du directeur des musées nationaux au directeur général des Beaux-Arts, 9 décembre 1935, AMN, P2R1A.

48. Résultats de la $1^{\text {re }}$ partie du Concours des restaurateurs, AMN, O5E. Le rentoileur Gaston Chauffrey est invité au jury de cette épreuve.

49. Idem.

50. $2^{\mathrm{e}}$ partie du Concours des restaurateurs, AMN, O5E. 
est la copie ${ }^{51}$ ", ou de Jean-Gabriel Goulinat, pour qui « le bon restaurateur doit posséder un talent de peintre ${ }^{52}$ ».
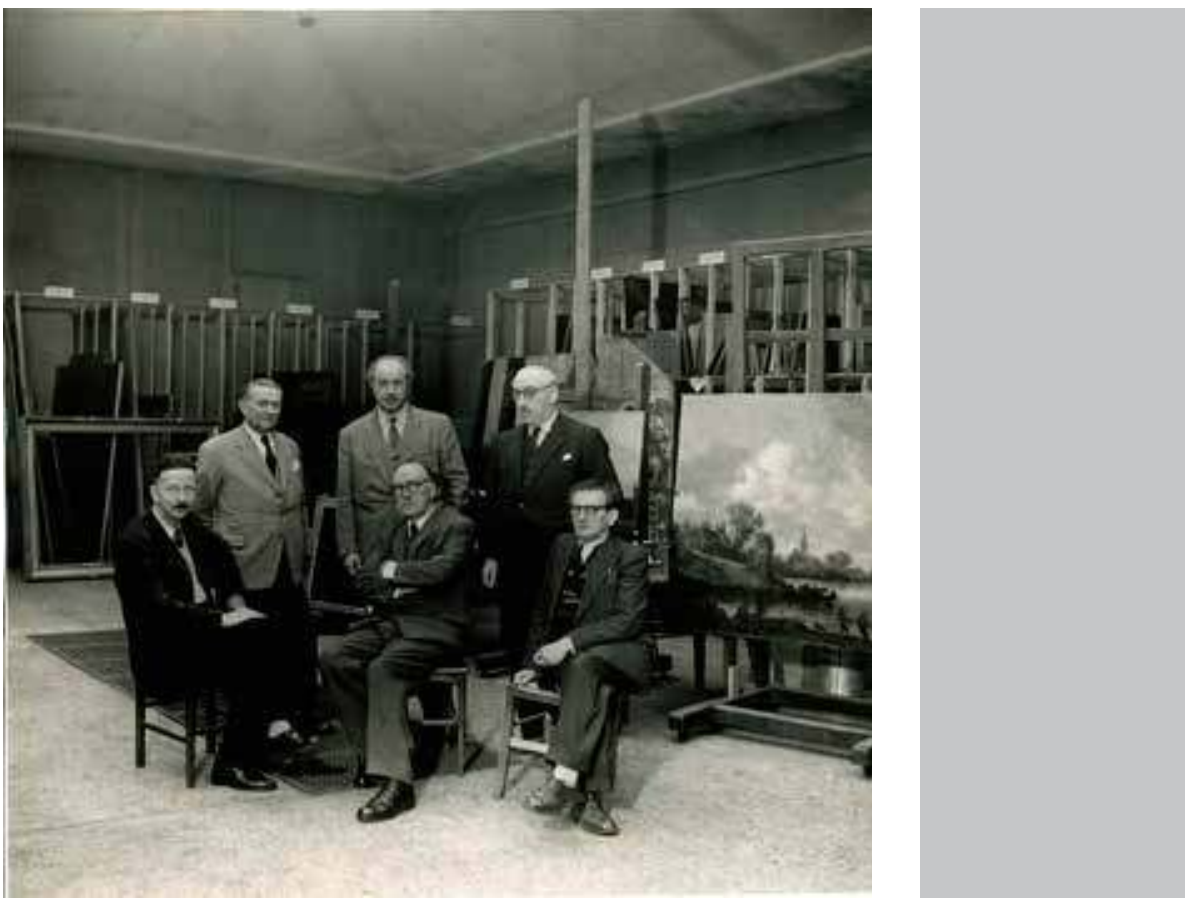

C'est le 26 novembre $1935^{53}$ que le directeur général des Beaux-Arts, suivant l'avis du jury ${ }^{54}$, signe l'arrêté par lequel les restaurateurs René Longa, Pierre Michel, Pierre Paulet et Georges Zezzos «sont nommés agréés par les musées nationaux ». Précédemment, Jean-Gabriel Goulinat avait été désigné par arrêté du $1^{\text {er }}$ juillet 1935 « chef d'atelier de restauration des peintures des musées nationaux, sous la direction du conservateur du département des peintures, des dessins et

Fig. 3

Les membres de l'atelier de restauration en 1949.

De gauche à droite, au premier plan : Lucien Aubert, Jean-Gabriel Goulinat, Georges Zezzos : au second plan : Edgard Aillet, Pierre Paulet, (C) Archives C2RMF, photo F. Parnotte

de la chalcographie du musée du Louvre ${ }^{55}$ ". Parmi les autres restaurateurs qui collaboraient avec les musées nationaux avant 1935, Edgard Aillet et Lucien Aubert sont désignés d'office pour travailler au sein du nouvel atelier, ce dernier conserve, en raison de son expérience, une place prééminente aux côtés de Jean-Gabriel Goulinat (fig. 3). On notera que le concours ne concerne pas les rentoileurs, le musée continuant à faire appel à des ateliers externes, principalement la maison Henry Leguay, devenue par la suite maison Trinquier et Léon Gard, qui ne semble pas avoir travaillé pour le Louvre après 1939, les rentoileurs Gaston Chauffrey et Marc-Rodolphe Muller ${ }^{56}$, qui s'associent à partir de 1945, et, ultérieurement, le rentoileur Raymond Lepage.

\section{L'activité de l'atelier de restauration des peintures des musées nationaux entre 1936 et 1950}

Le début des campagnes de restauration au musée du Louvre est marqué par l'arrivée de René Huyghe à la tête du département des Peintures en 1936,

51. Rapport adressé au directeur des musées nationaux concernant la restauration des tableaux du Louvre, 9 février 1932, AMN, P2 R30 Jacques Maroger.

52. Réunion de la Commission d'experts pour la conservation des peintures, 30-31 mars 1933, Archives de l'Unesco, OIM/VI/15.

53. Arrêté du 26 novembre 1935, AMN, O5E, Résultats définitifs du Concours des restaurateurs. 54. Idem, procès-verbal du 21 octobre 1935

55. AMN, O30 547. Cité par I. Cabillic, 2008, op. cit. note 6, p. 93.

56. Odette Berthelé, L'Atelier Chauffrey-Müller : 36 ans de, rentoilage et de restauration de peintures à Paris (1919-1955), mémoire de recherche, Paris, Ecole du Louvre, dir. Nathalie Volle, Clémence Raynaud, 2009 ; Emmanuelle Polack, Odette Berthelé, « Histoire de la restauration des fresques de l'église de Nohant-Vic et de leur copie au musée des Monuments français », Techné, n० 33,2011 , pp. 96-103. 
puis celle de Germain Bazin, nommé adjoint l'année suivante, et chargé plus particulièrement de la supervision des travaux de restauration ${ }^{57}$.

Un recensement des œuvres restaurées témoigne de l'intense activité au sein de l'atelier durant cette période ${ }^{58}$. Plus de 600 œuvres ont été restaurées, certaines à plusieurs reprises, dont 582 ont pu être identifiées. Il en ressort que le musée du Louvre est le principal bénéficiaire des travaux : 86,5\% des tableaux lui appartiennent, suivi de ceux du château de Versailles (4,5\%). Seulement 78,5 \% des peintures sont soumises à l'accord de la commission avant restauration, qui n'en contrôle que $12,4 \%$ après restauration.

Hormis les restaurations jugées urgentes en raison de l'état de conservation de certains tableau ${ }^{59}$, la programmation annuelle des travaux a suivi une organisation méthodique. Les années 1936 et 1937 sont principalement consacrées à une campagne de "dépoussiérage, nettoyage superficiel, et régénération des vernis " ${ }^{60}$ de plus de 300 tableaux exposés au Louvre, qualifiée de " véritable sauvetage ${ }^{61}$ ". Par la suite, les tableaux accrochés dans chaque salle sont abordés suivant le plan de fermeture progressive des salles d'exposition prévue par le plan Verne. La Seconde Guerre mondiale n'empêche pas la poursuite des restaurations, malgré des conditions de vie et de travail difficiles. Des antennes de l'atelier sont installées aux châteaux de La Pelice et de Sourches, ainsi qu'au collège Saint-Théodard et à l'Institut Calvin de Montauban où près de 150 interventions ont été recensées ${ }^{62}$.

Concernant la typologie des interventions, $18,5 \%$ des tableaux font l'objet d'un refixage de la couche picturale, $17,5 \%$ subissent une transposition et $7 \%$ un rentoilage. La décision est prise de ne pas intervenir sur $4 \%$ des œuvres présentées en commission, souvent en raison de leur fragilité. On sait également qu'une campagne de "stérilisation " de cadres menée par le laboratoire eut lieu en 1941, mais les sources d'archives ne donnent pas de précisions sur les objets concernés ${ }^{63}$.

L'intervention la plus courante à l'atelier de restauration est l'allégement des vernis dont il est possible d'affirmer qu'il est réalisé sur la grande majorité des tableaux restaurés, opération souvent suivie de la reprise des anciennes retouches. Ce procédé, vraisemblablement mis au point à partir de $1937^{64}$, consiste à enlever quelques couches des vernis anciens à l'aide de différents solvants sans atteindre le dévernissage complet. Il illustre la volonté de dépouiller les tableaux du « jus de musée " dont ils étaient recouverts, pratique au centre des débats doctrinaux opposant les musées nationaux français à certains musées allemands et anglosaxons partisans du dévernissage ${ }^{65}$. À ce propos, René Huyghe déclare : "Si nous prouvons que nous pouvons faire quelque chose de différent de ce qu'ils font et de mieux, nous renforcerons notre position ${ }^{66} "$.

57. Gilberte Émile-Mâle, « Germain Bazin (1901-1990) et la restauration des peintures au musée du Louvre », Coré, n 11, 2001, pp. 52-56 ; Magali Botlan, Germain Bazin et la restauration des peintures (1937-1965), mémoire d'étude, Paris, École du Louvre, dir. Nathalie Volle, Christophe Pincemaille, 2002.

58. Réalisé d'après les procès-verbaux des commissions de restauration et des devis et mémoires présentés par les restaurateurs. Ce recensement ne se prétend pas exhaustif, il est particulièrement lacunaire pour la période de la Seconde Guerre mondiale. Voir Fernando Suárez San Pablo, L'atelier de restauration des peintures des musées nationaux (1935-1950): Histoire, procédés et méthodes, mémoire de recherche, Paris, École du Louvre, sous la dir. de Clémence Raynaud, 2013, vol. 2, pp. 31-73.

59. Procès-verbal de la commission du 11 mai 1938, C2RMF.

60. D'après l'expression employée par les restaurateurs dans leurs devis et mémoires, conservés au C2RMF et aux AMN.

61. «Direction des musées nationaux - Diverses notes administratives 1938-1939, Projet de Budget, Chapitre 37 - musées nationaux - Matériel », AMN, O1A.

62. Isabelle Cabillic, «L'atelier de restauration de peintures du musée du Louvre pendant la Seconde Guerre mondiale », Technè, n० 33, 2011, pp. 53-60 ; voir aussi : Sabine Le Creff, Marguerite Sido, Conservation et restauration des tableaux du Louvre de 1939 à 1946, mémoire d'étude, Paris, Ecole du Louvre, dir. Nathalie Volle, Christophe Pincemaille, 2000; Amandine de Pérignon, La Conservation et la restauration de la collection de peinture du musée du Louvre pendant la Seconde Guerre mondiale au dépôt de Montauban, mémoire d'étude, Paris, École du Louvre, dir. N. Volle, I. Cabillic, 2006.

63. Comme en témoignent plusieurs pièces dans AMN, PC2.

64. Lettre de René Huyghe à Jean-Gabriel Goulinat, 20 octobre 1937, C2RMF, dossier CI2. Nous remercions Joëlle Cretin de nous avoir communiqué ces documents.

65. G. Bazin, cité par I. Cabillic, op. cit. note 62, p. 53.

66. Procès-verbal de la commission du 28 mars 1950, C2RMF. 
Parallèlement aux campagnes de nettoyage et de restauration apparait l'idée de lutter contre les causes de dégradation des œuvres d'art. En 1932, Jacques Maroger professe : "La vraie conservation doit prévenir la restauration. Il faut donc supprimer les causes d'intervention ${ }^{67}$ ", tandis qu'un document de l'administration constate en 1938 "qu'une grande partie des dépenses très lourdes des réparations actuelles aurait pu être évitée si les musées nationaux avaient disposé chaque année des crédits nécessaires à un entretien courant, beaucoup moins dispendieux, qui eût prévenu certaines altérations aggravées par le temps ${ }^{68}$. " En février 1931, le laboratoire étudie le climat des salles de peinture du Louvre, dont l'intérêt pour la conservation des œuvres avait déjà été souligné par Jean-François Cellerier ${ }^{69}$. Si cette question préoccupe toujours les conservateurs du Louvre, leurs essais se heurtent au manque de moyens de contrôle des conditions thermohygrométriques des salles autres que la régulation du système de chauffage, qui se montre insuffisant ${ }^{70}$. C'est avec plus de succès qu'un service "d'entretien" des peintures est établi en 1938. Le lundi, jour de fermeture du musée au public, deux restaurateurs, alternant par roulement hebdomadaire, réalisent des opérations de "nettoyage superficiel, dépoussiérage, traitement du vernis et menues restaurations ${ }^{71}$ ": "Ces soins préventifs donnés à temps éviteront certainement dans l'avenir les travaux toujours délicats et trop nombreux qui doivent être exécutés d'urgence quand le mal a déjà fait son œuvre néfaste ${ }^{72}$ ", conclue un rapport.

\section{Le plan Verne comme facteur de changement}

L'évolution de la politique de restauration des peintures des musées nationaux ne peut être entièrement comprise sans tenir compte des répercussions du plan de réaménagement du musée du Louvre entrepris par Henri Verne. En effet, c'est dans le cadre de la réorganisation du département des Peintures du musée du Louvre qu'on décide de restaurer systématiquement les collections.

Deux autres facteurs dérivés du plan Verne contribuent à l'ampleur et au succès des campagnes de restauration menées entre 1936 et 1950 . D’abord, les travaux ont pu être engagés grâce aux crédits extraordinaires reçus au titre des plans d' " outillage national ", dont une partie est destinée au financement des restauration ${ }^{73}$. Ainsi, le plan d'Adrien Marquet attribue à la restauration des peintures du musée du Louvre 250000 francs en 1937, la même somme l'année suivante, à laquelle s'ajoutent 98761 francs pour la restauration de tableaux du château de Versailles ${ }^{74}$. Ne disposant plus de cette manne en 1939, le Louvre ne semble disposer que de 100000 francs, dont 20000 francs sont réservés au programme de nettoyage hebdomadaire ${ }^{75}$. Le suivi des budgets disponibles durant la guerre est lacunaire. On sait cependant que 80000 francs sont affectés aux restaurations conduites au château de Sourches en 1940, et que 450000 francs ont été destinés en octobre 1942 aux rentoilages réalisés à Sourches et Montauban ${ }^{76}$. En 1947 le budget disponible est de 4000000 de francs, somme réduite à 2500000 francs l'année suivante, "ce qui, avec l'augmentation des prix, permettra d'effectuer moins de travaux » se plaint Germain Bazin ${ }^{77}$.

\footnotetext{
67. Op. cit. note 51 .

68. Op. cit. note 61 .

69. Copie du procès-verbal du Laboratoire d'Essais des Arts et Métiers au sujet de la détermination des températures et de l'état hygrométrique de l'air dans diverses salles du Musée, 2 avril 1931, AMN, PL2.

70. Comme en témoignent plusieurs pièces de Germain Bazin et René Huyghe dans AMN, 6LL3 1937-1949 (m).

71. Op. cit. note 59 .

72. Rapport sur l'administration et la conservation des musées nationaux pendant l'année 1936 et pendant l'année 1937 (janvier - octobre). Extrait du Journal Officiel de la République Française, 31 juillet, AMN, O2A ; procès-verbal de la commission du 23 janvier 1939, C2RMF.

73. H. Verne, 1934, op. cit. note 10, p. 9. Les sources d'archives parlent indistinctement $\mathrm{d}^{\prime} \ll$ outillage national » et de « plan Marquet ».

74. AMN, P2R5, plusieurs pièces.

75. Op. cit. note 61 .

76. I. Cabillic, op. cit. note 62, p. 59.

77. Procès-verbal de la commission du 9 juin 1949, C2RMF. Le budget de 1947 est dépensé en 1948, celui de 1948 en 1949. L'interprétation de ces sommes doit cependant tenir compte des forts taux d'inflation et des successives dévaluations ayant eu lieu de 1939 à 1948, liées à la Seconde Guerre mondiale.
} 
Par ailleurs, les travaux de réaménagement prévoient la fermeture des salles d'exposition du département des Peintures de manière graduelle entre 1937 et $1947^{78}$ (fig. 4). Cela a permis aux restaurateurs de travailler éloignés du regard du public et d'éviter la confrontation de tableaux présentés sous divers états de conservation et de nettoyage, ce qui aurait pu susciter l'incompréhension des amateurs. Un article paru dans La Croix le 15 juillet 1945, après la réouverture du Louvre, témoigne de la réception des restaurations, majoritairement saluée par la presse (fig. 5) : "La rencontre est émouvante. [...] Une surprise : la vivacité des couleurs. On a fort habilement fait la toilette des toiles, dont une poussière vénérable faisait oublier l'éternelle jeunesse qu'est celle des chefs-d'œuvre ${ }^{79}$ ».
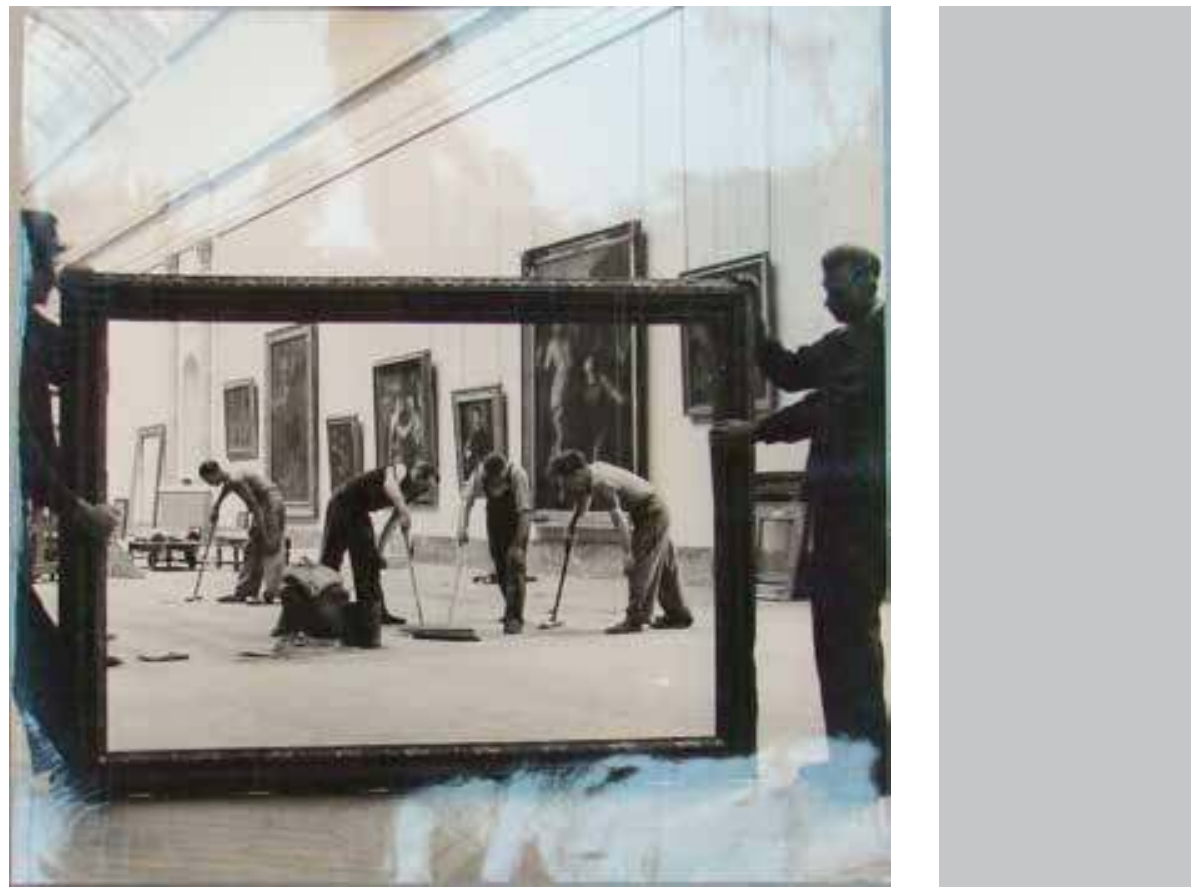

De 1930 à 1950, les musées nationaux ont développé un système d'organisation et de contrôle de la restauration des peintures qui répond aux préoccupations partagées par les institutions muséales rassemblées au sein de l'OIM. Multiples acteurs - conservateurs, scientifiques, restaurateurs et représentants de l'administration ont travaillé de concert à la définition d'une politique marquée par la prudence. Ainsi, la campagne de travaux pour présenter les collections de peinture du musée du Louvre dans un état de conservation en accord aux principes muséographiques modernes n'est entamée qu'après la définition d'un ensemble de principes sur la restauration, l'accord sur les méthodes de nettoyage, et la mise en place d'une nouvelle commission de restauration servant d'organe de contrôle. C'est dans ce contexte que, faute d'avoir développé une véritable théorie de la restauration, la mise au point du procédé d'allégement du vernis à permis aux musées nationaux français, dès 1948 , de prendre part sur le plan international aux débats de la "querelle des vernis ". Il doit être souligné le rôle essentiel joué par Henri Verne dans l'organisation de l'atelier de restauration des peintures des musées nationaux, ainsi que celui des conservateurs ayant mis en œuvre sa politique, René Huyghe auquel succède Germain Bazin, en 1950.

78. Déclarations de René Huyghe dans Luc Decaunes, «Musées : Jardins publics de l'art », Regards, 21 janvier 1937. Une copie de cet article est conservée dans AMN, O30 358.

79. Une collection des coupures de presse relative à la réouverture du musée, dont cet article, est conservée dans AMN, O30 358, « Coupures de presse 1945 ». 


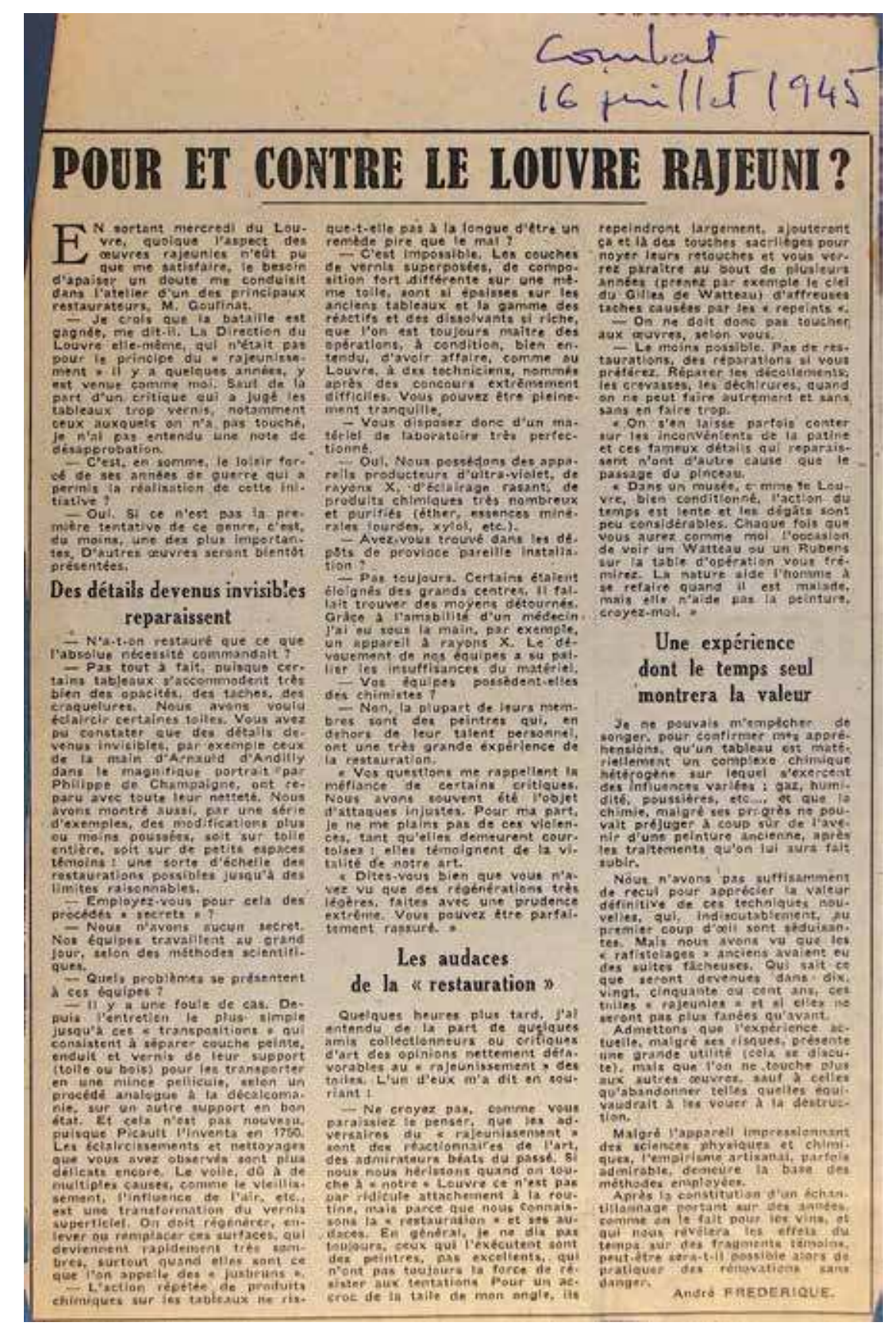

Fig. 5

André Frédérique

«Pour et contre le Louvre rajeuni? ? Combat, 16 juillet 1945
AMN, O30 358 (c) Archives nationales de Pierrefitte-sur-Seine 


\section{L'auteur}

Diplômé en histoire de l'art à l'Universidad Autónoma de Madrid (licence), Fernando Suárez San Pablo a soutenu un mémoire de Master 1 à l'École du Louvre consacré au restaurateur Pierre Michel, sous la direction de François Mirambet et d'Isabelle Cabillic. En 2013 il a soutenu un mémoire de recherche sous la direction de Clémence Raynaud intitulé L'atelier de restauration des peintures des musées Nationaux (1935-1950): Histoire, procédés et méthodes. 